\title{
Onset of Marangoni instability of a two-component evaporating droplet
}

\author{
Vai-Meng Ha, Chun-Liang Lai * \\ Department of Mechanical Engineering, National Taiwan University, Taipei 106, Taiwan, ROC
}

Received 30 December 2001; received in revised form 14 June 2002

\begin{abstract}
The temperature and solute concentration reductions across a thin boundary layer near the free surface of an evaporating droplet may induce cellular flow motion in the droplet because of Marangoni instability. The present study is aimed at investigating theoretically the onset of Marangoni instability due to the evaporation of a two-component evaporating droplet.

With the quasi-steady approximation which means that the surrounding gas motion is asymptotically steady, the size change of the droplet is negligible, and the temperature and concentration distributions of the droplet are temporarily frozen at each specified instant of interest, the onset condition for Marangoni instability is obtained through the linear stability analysis.

By assuming the surface tension is a monotonically decreasing function of both temperature and concentration of the higher-volatility substance, the thermocapillary and diffuso-capillary effects augment each other. Therefore, the theoretical analysis predicts a linear relation, with a negative slope, between the onset thermal Marangoni number, $M a_{\mathrm{T}}$, and the onset solute Marangoni number, $M a_{\mathrm{s}}$. Moreover, when liquid Lewis number $L e_{1}>1$, the critical wave number, $l_{\mathrm{c}}$, may possess different values depending on the variation of the thermocapillary effect and diffuso-capillary effect. In addition, $L e_{1}$ has a stronger effect on the critical solute Marangoni number $M a_{\mathrm{S}, \mathrm{C}}$, than on the critical thermal Marangoni number $M a_{\mathrm{T}, \mathrm{C}}$. That is, as $L e_{1}$ decreases, $M a_{\mathrm{T}, \mathrm{C}}$ decreases mildly while $M a_{\mathrm{S}, \mathrm{C}}$ increases drastically.

(c) 2002 Elsevier Science Ltd. All rights reserved.
\end{abstract}

Keywords: Marangoni instability; Evaporation; Thermocapillary; Diffuso-capillary

\section{Introduction}

When there is a temperature or concentration gradient along the interface or free surface, flow in the liquid phase will be generated immediately by the thermocapillary or diffuso-capillary effect. Such a flow motion is called thermocapillary flow or diffuso-capillary flow. If the temperature or concentration gradient is normal to the interface or free surface, cellular flow motion will be generated only when the corresponding Marangoni number exceeds a certain critical value. Such a flow phenomenon is called Marangoni instability.

The instability of a liquid layer heated from below was first studied by Pearson [23]. Since then, Marangoni instability due solely to a temperature gradient $[8,11-13,18,20,26,28,31-33]$ or concentration gradient $[3,9,21,29]$ has been extensively studied. Among them, Nield [20], Smith [28] and Davis [8] included also the buoyancy effect. Marangoni instability with a deformable surface were investigated by Scriven and Sternling [26], Zeren and Reynolds [33], Wahal and Bose [32], and Ha [11]. Recently, a few studies of a vaporizing droplet with surface tension effect have appeared

\footnotetext{
${ }^{*}$ Corresponding author. Tel.: +886-02-2363-4820; fax: +886-02-2363-1755.

E-mail address: cllai@w3.me.ntu.edu.tw (C.-L. Lai).
} 


\begin{tabular}{|c|c|c|c|}
\hline \multicolumn{4}{|c|}{ Nomenclature } \\
\hline$A_{0}$ & gas evaporating velocity at the droplet sur- & $Y_{l}^{m}$ & spherical harmonics \\
\hline & face & $\mathbf{Y}$ & magnitude of mass fraction perturbation \\
\hline a & activity of the volatile component & \multicolumn{2}{|c|}{ Greek symbols } \\
\hline$B i$ & heat exchange parameter & $\alpha$ & thermal diffusivity \\
\hline$C_{p}$ & heat capacity & $\gamma$ & ratio of the activity of the volatile compo \\
\hline $\begin{array}{ll}C r \\
D\end{array}$ & Crispation number & & nent to its surface mole fraction \\
\hline $\begin{array}{l}D \\
\text { Ev }\end{array}$ & $\begin{array}{l}\text { mass diffusivity } \\
\text { dimensionless evaporation rate }\end{array}$ & $\zeta_{1}$ & defined parameter indicating the mass \\
\hline$h_{\mathrm{lg}}$ & $\begin{array}{l}\text { latent heat of evaporation related to the } \\
\text { concentration gradient }\end{array}$ & $\zeta_{2}$ & $\begin{array}{l}\text { transfer due to temperature perturbations } \\
\text { defined parameter indicating the mass }\end{array}$ \\
\hline$k$ & thermal conductivity & & transfer due to concentration perturbations \\
\hline Le & Lewis number & $\mu$ & dynamic viscosity \\
\hline$l$ & wave number of the disturbances & $v$ & kinematic viscosity \\
\hline$M a$ & Marangoni number & $\rho$ & density \\
\hline$M_{\mathrm{amb}}$ & molecular weight of air & & surface tension \\
\hline$M_{11}$ & molecular weight of the volatile component & & dimensionless time \\
\hline$M_{12}$ & $\begin{array}{l}\text { molecular weight of the non-volatile com- } \\
\text { ponent }\end{array}$ & $\begin{array}{l}\varphi_{k} \\
\phi_{\alpha}\end{array}$ & thermal diffusivity ratio \\
\hline$m$ & azimuthal wave number & $\varphi_{\rho}$ & $\begin{array}{l}\text { density ratio } \\
\text { defined parameter indicating the he }\end{array}$ \\
\hline$m^{\prime}$ & dimensional evaporation rate & & $\begin{array}{l}\text { transfer due to temperature perturbation } \\
\text { tom }\end{array}$ \\
\hline$m_{\mathrm{v}}$ & $\begin{array}{l}\text { vapor mole fraction of the volatile compo- } \\
\text { nent }\end{array}$ & $\chi_{2}$ & defined parameter indicating the hea \\
\hline $\operatorname{Pr}$ & Prandtl number & $(1)$ & complex quantity representing the time \\
\hline$P_{l}^{m}$ & associated Legendre polynomials & $\omega$ & varying behavior of disturbances \\
\hline$p$ & $\begin{array}{l}\text { pressure } \\
\text { dimensionless heat flux due to the evapora- }\end{array}$ & $\omega_{i}$ & circular frequency of the disturbance \\
\hline$Q$ & $\begin{array}{l}\text { dimensionless heat flux due to the evapora- } \\
\text { tion }\end{array}$ & $\omega_{r}$ & amplification factor of the disturbance \\
\hline$R, \theta, \phi$ & dimensionless spherical coordinates & $M i$ & neous symbols \\
\hline $\operatorname{Re}$ & radial Reynolds number of the gas phase & $l$ & imaginary unit \\
\hline & flow & $\mathfrak{R}$ & universal gas constant \\
\hline$r, \theta, \phi$ & dimensional spherical coordinates & $\mathfrak{I}$ & magnitude of temperature perturbation \\
\hline$r_{0}$ & radius of the evaporating droplet & & ipts \\
\hline$r_{\mathrm{w} 1}$ & ratio of molecular weights $M_{\mathrm{amb}} / M_{11}$ & & perturbed quantity \\
\hline $\begin{aligned} r_{\mathrm{w} 2} \\
\dot{\boldsymbol{c}}\end{aligned}$ & ratio of molecular weights $M_{12} / M_{11}$ & * & dimensionless quantity \\
\hline & rating free surface & & \\
\hline$T$ & temperature & $\mathrm{b}$ & boiling state \\
\hline$t$ & dimensional time & $\mathrm{g}$ & gas phase \\
\hline$U$ & magnitude of the perturbed $R$-component & 1 & liquid phase \\
\hline & velocity & $\mathrm{v}$ & vapor (of the volatile component) \\
\hline$\vec{V}$ & velocity vector & ref & reference state \\
\hline$x$ & surface liquid mole fraction of the volatile & $\mathrm{s}$ & surface condition \\
\hline & component & 0 & initial condition \\
\hline$Y$ & mass fraction of the volatile component & $\infty$ & far field \\
\hline
\end{tabular}

$[15,19,25]$. Generally, linear stability analysis was applied mostly in the past. As an alternative approach, the energy method was proposed and applied by Davis [8], Homsy [13], and Neitzel [18].

There are some physical systems, such as the evaporation of a liquid solution, that simultaneous thermal and concentration gradients may exist in the liquid. If there is an interface, Marangoni instability may occur due to the coupling effect of the non-uniform surface temperature and concentration distributions.

McTaggart [17] has studied the Marangoni instability of a liquid layer with both temperature and concentration gradients in the absence of gravity. She found that the surface tension effects due to these two gradients were additive. 
Moreover, for the aqueous solution of $\mathrm{MgSO}_{4}$, there existed a case that oscillatory instability became the preferred mode. Slavtchev et al. [27] investigated the stationary Marangoni instability in a liquid layer due to non-isothermal gas absorption. Instability was found for various conditions.

Experiments on the Marangoni instability of three different aqueous solutions were studied by Chen [4]. Linear stability analysis was applied by Chen and $\mathrm{Su}[5]$ to study a similar problem. The results showed that, with or without buoyancy effect, the predicted stability boundaries were quite different. When the surface tension effect and the buoyancy effect opposed each other, the oscillatory instability became the preferred mode. Moreover, a bimodal marginal instability curves could be generated, which means that simultaneous occurrence of two instability modes with different wave numbers were possible. Tanny et al. [30] modified the work of Chen and Su [5] to further study the effect of nonlinear concentration distribution across the layer depth both experimentally and by linear stability analysis. The theoretical results were in reasonable agreement with the experiments.

The above studies, however, have not actually considered the effects of phase change, surface regression, or transient energy and species diffusion. Insulated and non-diffusive boundary conditions were usually applied at the free surface. Actually, the continuity conditions of the heat flux, mass flux, velocity and stresses should be applied at the interface. Recently, Aharon and Shaw [1,2] employed the linear stability analysis to study the onset conditions of stationary Marangoni instability of an evaporating bi-component droplet with surface regression. Only quasi-steady species profiles with spherical symmetry were considered in their study. In addition to the droplet, the surrounding gas phase was also perturbed and coupled with the liquid phase. Critical radii for stability of two different liquid mixtures were then determined. However, evaporation is an unsteady process involving simultaneous heat and mass transports. The heat transfer rate of the droplet is mainly controlled by the mass flux at the free surface during evaporation. There exists a strong coupling between the temperature and concentration fields. Therefore, the temperature variation of the droplet during evaporation should not be ignored.

In order to complement the work by Aharon and Shaw [1,2] the coupling effects upon the onset of Marangoni instability of an evaporating bi-component droplet due to simultaneous temperature and concentration variations are investigated in the present study. Under the quasi-steady assumption, which will be discussed in next section, the onset conditions of stationary Marangoni instability are obtained analytically by linear stability analysis. The relation between the onset thermal Marangoni number and the onset solute Marangoni number and the mechanism for the onset of Marangoni instability are clearly and explicitly demonstrated.

\section{Mathematical formulation}

A motionless droplet of radius $r_{0}$ surrounded by a passive gas with an initial mass fraction $Y_{\infty}$ of the evaporating component and at temperature $T_{0}$ and pressure $p_{\infty}$ is considered in this study. The droplet is originally at pressure $p_{0}$ and the same initial temperature $T_{0}$ as the surrounding gas. $Y_{0}$ is the mass fraction of the volatile component of the droplet. A schematic diagram of the physical model is depicted in Fig. 1.

\subsection{Basic assumptions}

\subsubsection{Quasi-steady approximation}

Strictly speaking, evaporation of a droplet is never a steady process since the radius of the droplet, the surface temperature, and the evaporation rate keep decreasing during evaporation. At distances far from the droplet surface, the unsteady effect in the gas phase might be important [6]. However, as shown in Table 1, the time scales of the gas

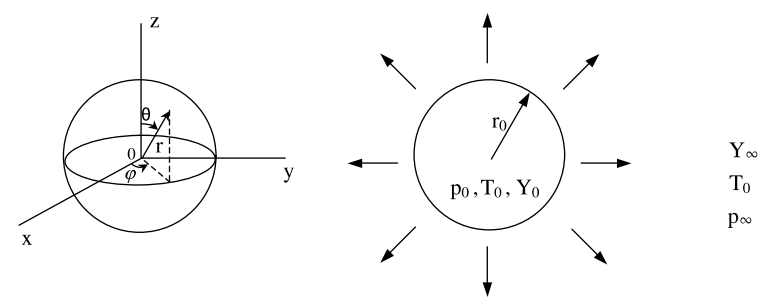

Fig. 1. The schematic diagram of the physical model and coordinate system. 
Table 1

Comparison of the various timescales during evaporation: $\alpha_{1} / \alpha_{\mathrm{g}} \sim \mathrm{O}\left(10^{-2}\right), \rho_{1} / \rho_{\mathrm{g}} \sim \mathrm{O}\left(10^{3}\right), \mu_{1} / \mu_{\mathrm{g}} \sim \mathrm{O}\left(10^{2}\right), \operatorname{Pr} \sim \mathrm{O}\left(10^{1}\right), \operatorname{Pr}_{\mathrm{g}} \sim 1$, $L e_{1} \sim\left(10^{1} \sim 10^{2}\right), L e_{\mathrm{g}} \sim\left(10^{-1} \sim 1\right), \quad R e=A_{0} r_{0} / v_{\mathrm{g}}=$ radial Reynolds number $\sim \mathrm{O}\left(10^{-1} \sim 1\right), A_{0}=\left.u_{r, \mathrm{~g}}\right|_{r=r_{0}}=-\frac{D_{\mathrm{g}}}{r_{0}} \ln \frac{1-Y_{\mathrm{v}, 0}(1)}{1-Y_{\mathrm{v}, 0}(1) / \phi_{\rho}}=\operatorname{gas}$ evaporating velocity at the droplet surface

\begin{tabular}{lll}
\hline Process & Timescale & Timescale w.r.t. $r_{0}^{2} / \alpha_{1}$ \\
\hline Gas phase flow & $r_{0} / A_{0}$ & $\frac{\alpha_{1}}{A_{0} r_{0}}=\frac{1}{R e P_{1}} \frac{\mu_{1}}{\mu_{\mathrm{g}}} \frac{\rho_{\mathrm{g}}}{\rho_{1}} \sim\left(10^{-2} \sim 10^{-1}\right)$ \\
Transport in gas phase & $r_{0}^{2} / v_{\mathrm{g}}$ & $\frac{\alpha_{\mathrm{l}}}{v_{\mathrm{g}}}=\frac{\alpha_{1}}{\alpha_{\mathrm{g}}} \frac{1}{P r_{\mathrm{g}}} \sim 10^{-2}$ \\
(a) Diffusion of momentum & $r_{0}^{2} / \alpha_{\mathrm{g}}$ & $\frac{\alpha_{1}}{\alpha_{\mathrm{g}}} \sim 10^{-2}$ \\
(b) Diffusion of heat & $r_{0}^{2} / D_{\mathrm{g}}$ & $\frac{\alpha_{1}}{D_{\mathrm{g}}}=\frac{\alpha_{1}}{\alpha_{\mathrm{g}}} L e_{\mathrm{g}} \sim\left(10^{-3} \sim 10^{-2}\right)$ \\
(c) Diffusion of mass & & \\
Transport in liquid phase & $r_{0}^{2} / v_{1}$ & $\frac{\alpha_{1}}{v_{1}}=\frac{1}{P_{r_{1}}} \sim 10^{-1}$ \\
(a) Diffusion of momentum & $r_{0}^{2} / \alpha_{1}$ & 1 \\
(b) Diffusion of heat & $r_{0}^{2} / D_{1}$ & $\frac{\alpha_{1}}{D_{1}}=L e_{1} \sim\left(10^{1} \sim 10^{2}\right)$ \\
(c) Diffusion of mass & $\frac{r_{0}}{\dot{r}_{0}} \simeq \frac{r_{0} \rho_{1}}{A_{0} \rho_{\mathrm{g}}}$ & $\frac{\rho_{\alpha_{1}}}{A_{0} r_{0}} \frac{\rho_{\mathrm{g}}}{\rho_{\mathrm{g}}}=\frac{1}{R e P_{1}} \frac{\mu_{1}}{\mu_{\mathrm{g}}} \sim\left(10^{1} \sim 10^{2}\right)$ \\
Droplet regression & & \\
\hline
\end{tabular}

phase are two order smaller than that of the heat diffusion in the liquid phase. Moreover, the state of the evaporating droplet is far below the critical state of phase change. Therefore, the gas phase (the surrounding air) can be treated as asymptotically steady. On the other hand, the time scale for the interface regression is about one order larger than the heat diffusion time scale of the liquid phase. The regression of the droplet surface is, therefore, negligible when consideration is mainly on the droplet temperature evolution. Although the time scale of the surface regression is about the same order as the time scale of species diffusion in the droplet, the surface regression is still neglected in the present study. The reasons are as follows: (1) Since all the Marangoni instabilities occur at the very early stage during evaporation, the surface regression is extremely small and, therefore, can be neglected. (2) The unsteady term in species diffusion equation is purposely included so that its effect on the Marangoni instability due to evaporation can be studied; its inclusion does not improve the accuracy of the analysis. Therefore, the present study is limited to the investigation of how the Marangoni instability of an evaporating droplet, which is mainly driven by the thermocapillary effect, is influenced or modified by the diffuso-capillary effect at the very early stage during evaporation when the surface regression can be neglected.

Moreover, because the distributions of the temperature and species concentration of the liquid phase vary continuously during evaporation, it is difficult to deal with the onset of Marangoni instability by linear analysis. In order to obtain basic ideas and sufficient conditions for the onset of instability, the temperature and concentration distributions are temporarily "frozen" at each specified instant during the application of linear stability analysis $[2,11,12]$.

The quasi-steady approximation used in the present study are summarized as follows:

1. The transport phenomena of the surrounding gas phase are treated as steady.

2. The regression of the interface during evaporation is negligibly small.

3. The temperature and species concentration distributions of the liquid phase are temporarily frozen during the linear stability analysis.

\subsubsection{General assumptions for simplification}

In addition to the quasi-steady approximation discussed above, the following assumptions are made to further simplify the analysis:

1. The instability of a binary droplet with one volatile component is considered.

2. The free surface is undeformable.

3. Both the liquid and gas phases are assumed incompressible.

4. The buoyancy effect is neglected under the microgravity condition.

5. All the physical properties of the fluid except the surface tension, which is a monotonically decreasing function of both temperature and concentration of the volatile component, are considered constant.

6. The differential vapour recoil mechanism [22] is not considered at the droplet surface.

7. Viscous dissipation and radiative heat transfer are neglected.

8. Under the assumption of thermodynamic equilibrium at the droplet surface, the following vapor-liquid relation for the volatile component of a binary droplet should be satisfied [24], 


$$
Y_{\mathrm{v}}\left(r_{0}\right)=\frac{\gamma F \frac{r_{\mathrm{w} 2} Y_{1}\left(r_{0}\right)}{1-Y_{1}\left(r_{0}\right)+r_{\mathrm{w} 2} Y_{1}\left(r_{0}\right)} \exp \left[\frac{h_{\mathrm{lg}}}{\mathfrak{R}}\left(\frac{1}{T_{\mathrm{b}}}-\frac{1}{T_{\mathrm{s}}}\right)\right]}{r_{\mathrm{w} 1}-\left(r_{\mathrm{w} 1}-1\right) \gamma F \frac{r_{\mathrm{w} 2} Y_{1}\left(r_{0}\right)}{1-Y_{1}\left(r_{0}\right)+r_{\mathrm{w} 2} Y_{1}\left(r_{0}\right)} \exp \left[\frac{h_{\mathrm{lg}}}{\mathfrak{R}}\left(\frac{1}{T_{\mathrm{b}}}-\frac{1}{T_{\mathrm{s}}}\right)\right]},
$$

where $Y_{\mathrm{v}}\left(r_{0}\right)$ and $Y_{1}\left(r_{0}\right)$ are the vapor and liquid mass fraction of the volatile component at the free surface. Activity coefficient $\gamma$ is defined as the ratio of the activity of the volatile component, a, to its surface mole fraction, $x$, i.e. $\gamma \equiv \mathbf{a} / x$. $\mathrm{F}$ is a correction factor and usually close to unity when the total pressure is not too high; here, its value is set to be unity. $h_{\mathrm{lg}}$ is the heat of evaporation, $T_{\mathrm{b}}$ is the boiling temperature of the volatile component, and $\mathfrak{R}$ is the universal gas constant. $r_{\mathrm{w} 1}$ is the ratio of molecular weight of the non-volatile component (with subscript " 2 ") to that of the volatile one (with subscript " 1 "), i.e., $M_{12} / M_{11} . r_{\mathrm{w} 2}$ is the ratio of molecular weight of the ambient gas to that of the volatile species, i.e., $M_{\mathrm{amb}} / M_{11}$.

\subsection{The governing equations and basic solutions}

For the convenience and generality of the analysis and discussion, the variables are non-dimensionalized. The reference scales for length, time, velocity, temperature, pressure and mass fraction are $r_{0}, r_{0}^{2} / \alpha_{1}, \alpha_{1} / r_{0}, m_{0}^{\prime} h_{\mathrm{lg}} r_{0} / k_{1}, \rho_{1} \alpha_{1}^{2} / r_{0}^{2}$, and $Y_{0}$, respectively. In which, $\alpha_{1}$ is the liquid thermal diffusivity, $k_{1}$ is the liquid thermal conductivity, $\rho_{1}$ is the liquid density, and $m_{0}^{\prime}=\rho_{\mathrm{g}}\left(\left.u_{r, \mathrm{~g}}\right|_{r=r_{0}}-\dot{r}_{0}\right)$ is the evaporating rate of the volatile component at the initial temperature $T_{0}$. By defining the dimensionless temperature (subscript $\mathrm{g}$ and 1 refer to the gas phase and liquid phase, respectively)

$$
T_{1}^{*}=\frac{T_{1}-T_{0}}{T_{\text {ref }}} \quad \text { and } \quad T_{\mathrm{g}}^{*}=\frac{T_{\mathrm{g}}-T_{0}}{T_{\text {ref }}}
$$

the dimensionless governing equations and initial and boundary conditions and the corresponding basic solutions are as follows.

\subsubsection{Gas phase (the surrounding atmosphere)}

The evaporation will result in an outward flow motion away from the droplet surface in the gas phase. The governing equations for such a radial gas flow are as follows:

$$
\begin{aligned}
& \frac{1}{R^{2}} \frac{\partial}{\partial R}\left(R^{2} u_{R, \mathrm{~g}}^{*}\right)=0 \\
& \frac{\phi_{\alpha}}{P r_{\mathrm{g}}}\left(u_{R, \mathrm{~g}}^{*} \frac{\partial u_{R, \mathrm{~g}}^{*}}{\partial R}+\phi_{\rho} \frac{\partial p_{\mathrm{g}}^{*}}{\partial R}\right)=\frac{1}{R^{2}} \frac{\partial}{\partial R}\left(R^{2} \frac{\partial u_{R, \mathrm{~g}}^{*}}{\partial R}\right)-\frac{2 u_{R, g}^{*}}{R^{2}} \\
& \phi_{\alpha} u_{R, g}^{*} \frac{\partial T_{\mathrm{g}}^{*}}{\partial R}=\frac{1}{R^{2}} \frac{\partial}{\partial R}\left(R^{2} \frac{\partial T_{\mathrm{g}}^{*}}{\partial R}\right) \\
& \phi_{\alpha} L e_{\mathrm{g}} u_{R, g}^{*} \frac{\partial Y_{\mathrm{v}}^{*}}{\partial R}=\frac{1}{R^{2}} \frac{\partial}{\partial R}\left(R^{2} \frac{\partial Y_{\mathrm{v}}^{*}}{\partial R}\right) .
\end{aligned}
$$

The boundary conditions as $R \rightarrow \infty$ are

$$
\begin{array}{ll}
\vec{V}_{\mathrm{g}}^{*}=0, & T_{\mathrm{g}}^{*}=0, \\
p_{\mathrm{g}}^{*}=p_{\infty}^{*}, & Y_{\mathrm{v}}^{*}=Y_{\infty}^{*}=0 .
\end{array}
$$

At the droplet surface $R=1$, one has

$$
\begin{aligned}
& u_{R, 1}^{*}-\dot{S}=\frac{1}{\phi_{\rho}}\left(u_{R, \mathrm{~g}}^{*}-\dot{S}\right)=\mathrm{Ev} \\
& \frac{1}{\phi_{\alpha} L e_{\mathrm{g}}} \frac{\partial Y_{\mathrm{v}}^{*}}{\partial R}=-\left(\frac{1}{Y_{0}}-Y_{\mathrm{v}}^{*}\right)\left(u_{R, g}^{*}-\dot{S}\right) .
\end{aligned}
$$

In the above equations, $u_{R}^{*}$ is the dimensionless $R$-component velocity; $p^{*}$ denotes the dimensionless pressure; $Y_{1}^{*}$ and $Y_{\mathrm{v}}^{*}$ are the dimensionless liquid and vapor mass fraction of the volatile component. The property ratios, $\phi_{\alpha}=\alpha_{\mathrm{l}} / \alpha_{\mathrm{g}}$ and $\phi_{\rho}=\rho_{\mathrm{l}} / \rho_{\mathrm{g}}$, are the thermal diffusivity and the density ratio, respectively. $P r_{\mathrm{g}}=v_{\mathrm{g}} / \alpha_{\mathrm{g}}$ is the gas Prandtl number. $L e_{\mathrm{g}}=\alpha_{\mathrm{g}} / D_{\mathrm{g}}$ denotes the gas Lewis number with $D_{\mathrm{g}}$ being the mass diffusivity. Ev $=m^{\prime} r_{0} /\left(\rho_{1} \alpha_{1}\right)$ is a dimensionless 
evaporation rate. $\dot{S}$ is the dimensionless regression rate of the droplet and is evaluated only when mass balance is considered. Nevertheless, the position change of the interface, i.e., the droplet regression, is to be neglected in the later analysis under the quasi-steady approximation.

The basic solutions are

$$
\begin{aligned}
& u_{R, \mathrm{~g}}^{*}=-\frac{1}{\phi_{\alpha} L e_{\mathrm{g}}} \ln \frac{1-Y_{\mathrm{v}}(1)}{1-\frac{Y_{\mathrm{v}}(1)}{\phi_{\rho}}} \cdot \frac{1}{R^{2}}, \\
& p_{\mathrm{g}}^{*}=-\frac{1}{2 \phi_{\alpha}^{2} \phi_{\rho} L e_{\mathrm{g}}^{2}} \ln ^{2} \frac{1-Y_{\mathrm{v}}(1)}{1-\frac{Y_{\mathrm{v}}(1)}{\phi_{\rho}}} \cdot \frac{1}{R^{4}}+p_{\infty}^{*}, \\
& T_{\mathrm{g}}^{*}=T_{\mathrm{s}}^{*} \frac{\left[\frac{1-Y_{\mathrm{v}}(1)}{1-\frac{Y_{\mathrm{v}}(1)}{\phi_{\rho}}}\right]^{1 /\left(L e_{\mathrm{g}} \cdot R\right)}-1}{\left[\frac{1-Y_{\mathrm{v}}(1)}{1-\frac{Y_{\mathrm{v}}(1)}{\phi_{\rho}}}\right]^{1 / L e_{\mathrm{g}}}-1}, \\
& Y_{\mathrm{v}}^{*}=\frac{Y_{\mathrm{v}}(1)}{Y_{0}} \frac{\left[\frac{1-Y_{\mathrm{v}}(1)}{1-\frac{Y_{\mathrm{v}}(1)}{\phi_{\rho}}}\right]^{1 / R}-1}{\left[\frac{1-Y_{\mathrm{V}}(1)}{1-\frac{Y_{\mathrm{V}}(1)}{\phi_{\rho}}}\right]-1},
\end{aligned}
$$

where

$$
Y_{\mathrm{v}}(1)=\frac{\gamma \frac{r_{\mathrm{w} 2} Y_{1}(1)}{1-Y_{1}(1)+r_{\mathrm{w} 2} Y_{1}(1)} \exp \left[\frac{h_{\mathrm{lg}}}{\Re}\left(\frac{1}{T_{\mathrm{b}}}-\frac{1}{T_{\mathrm{s}}}\right)\right]}{r_{\mathrm{w} 1}-\left(r_{\mathrm{w} 1}-1\right) \gamma \frac{r_{\mathrm{w}} Y_{1}(1)}{1-Y_{1}(1)+r_{\mathrm{w} 2} Y_{1}(1)} \exp \left[\frac{h_{\mathrm{lg}}}{\Re}\left(\frac{1}{T_{\mathrm{b}}}-\frac{1}{T_{\mathrm{s}}}\right)\right]}
$$

is the mass fraction of the vapor at the droplet surface.

The dimensionless evaporation rate is then determined by the continuity of mass flux at the free surface, i.e., Eq. $(11)$,

$$
\mathrm{Ev}=\frac{\left.u_{R, g}^{*}\right|_{R=1}}{\phi_{\rho}-1}=\frac{R e P r_{\mathrm{g}}}{\phi_{\alpha}\left(\phi_{\rho}-1\right)}=-\frac{1}{L e_{\mathrm{g}} \phi_{\alpha}\left(\phi_{\rho}-1\right)} \ln \frac{1-Y_{\mathrm{v}}(1)}{1-\frac{Y_{\mathrm{v}}(1)}{\phi_{\rho}}},
$$

where $R e=\left.u_{r, \mathrm{~g}}\right|_{r=r_{0}} r_{0} / v_{\mathrm{g}}=u_{R, \mathrm{~g}}^{*} \alpha_{1} / v_{\mathrm{g}}$ is the radial Reynolds number of the gas phase flow (please referred to Table 1).

\subsubsection{Liquid phase (the droplet)}

Since the attention is focused on the evaporation of a quiescent droplet, no motion occurs in the liquid phase. The pressure in the droplet is then constant and can be determined by the condition of normal-force balance on the surface,

$$
-p_{1}^{*}-\left(-p_{\mathrm{g}}^{*}+\frac{2 P r_{\mathrm{g}}}{\phi_{\alpha} \phi_{\rho}} \frac{\partial u_{R, \mathrm{~g}}^{*}}{\partial R}\right)=-2 \frac{P r_{1}}{C r},
$$

where $P r_{1}=v_{1} / \alpha_{1}$ is the liquid Prandtl number and $C r=\mu_{1} \alpha_{1} /\left(\sigma_{0} r_{0}\right)$ is the Crispation number with $\sigma_{0}$ being the surface tension at $T_{0}$ and $Y_{0}$. Then, with $u_{R, g}^{*}$ and $p_{\mathrm{g}}^{*}$ solved, $p_{1}^{*}$ is determined as follows:

$$
p_{1}^{*}=\frac{2 P r_{1}}{C r}-\frac{4 P r_{\mathrm{g}}}{\phi_{\alpha}^{2} \phi_{\rho} L e_{\mathrm{g}}} \ln \frac{1-Y_{\mathrm{v}}(1)}{1-\frac{Y_{\mathrm{v}}(1)}{\phi_{\rho}}}-\frac{1}{2 \phi_{\rho} \phi_{\alpha}^{2} L e_{\mathrm{g}}^{2}} \ln ^{2} \frac{1-Y_{\mathrm{v}}(1)}{1-\frac{Y_{\mathrm{v}}(1)}{\phi_{\rho}}}+p_{\infty}^{*} .
$$

The temperature field of the droplet, $T_{1}^{*}$, and the distribution of the mass fraction of the volatile component, $Y_{1}^{*}$, cannot be solved analytically. Numerical calculations have to be used to solve the following energy and diffusion equations with the relevant conditions:

$$
\begin{aligned}
& \frac{\partial T_{1}^{*}}{\partial \tau}=\frac{1}{R^{2}} \frac{\partial}{\partial R}\left(R^{2} \frac{\partial T_{1}^{*}}{\partial R}\right), \\
& L e_{1} \frac{\partial Y_{1}^{*}}{\partial \tau}=\frac{1}{R^{2}} \frac{\partial}{\partial R}\left(R^{2} \frac{\partial Y_{1}^{*}}{\partial R}\right),
\end{aligned}
$$




$$
\begin{aligned}
& \tau=0, \quad T_{1}^{*}=0, \quad Y_{1}^{*}=1, \\
& R=0, \quad T_{1}^{*}, Y_{1}^{*}=\text { finite }, \\
& R=1, \quad-\frac{\partial T_{1}^{*}}{\partial R}=T_{\mathrm{s}}^{*} B i+Q, \\
& -\frac{1}{L e_{1}} \frac{\partial Y_{1}^{*}}{\partial R}=-Y_{1}^{*} \mathrm{Ev}+\frac{\mathrm{Ev}}{Y_{0}} .
\end{aligned}
$$

In Eq. (27), the first term of the right-hand side represents the heat transfer to the ambient by convection, and the second term denotes the heat flux due to evaporation at the free surface. Relevant parameters are

$$
\begin{aligned}
& B i=\frac{1}{\phi_{k} L e_{\mathrm{g}}} \ln \frac{1-Y_{\mathrm{v}}(1)}{1-\frac{Y_{\mathrm{v}}(1)}{\phi_{\rho}}} \cdot \frac{\left[\frac{1-Y_{\mathrm{V}}(1)}{1-\frac{Y_{\mathrm{V}}(1)}{\phi_{\rho}}}\right]^{1 / L e_{\mathrm{g}}}}{\left[\frac{\left(1-Y_{\mathrm{V}}(1)\right.}{1-\frac{Y_{\mathrm{V}}(1)}{\phi_{\rho}}}\right]^{1 / L e_{\mathrm{g}}}-1}, \\
& Q=\frac{m^{\prime}}{m_{0}^{\prime}}=\frac{\rho_{1} \alpha_{1} \mathrm{Ev} / r_{0}}{\rho_{1} \alpha_{1} \mathrm{Ev} v_{0} / r_{0}}=\frac{\ln \frac{1-Y_{\mathrm{V}}(1)}{1-\frac{Y_{\mathrm{V}}(1)}{\phi_{\rho}}}}{\ln \frac{1-Y_{\mathrm{V}, 0}(1)}{1-\frac{Y_{\mathrm{V}}, 0(1)}{\phi_{\rho}}}},
\end{aligned}
$$

where $\phi_{k}=k_{1} / k_{\mathrm{g}}$ is the thermal conductivity ratio.

The transient temperature and concentration distributions of the droplet are solved numerically by using the CrankNicolson method, which is of second-order accuracy $\left[(\Delta \tau)^{2},(\Delta R)^{2}\right]$. To obtain a true transient solution, the quantities $\Delta \tau$ and $\Delta R$ have been chosen in such a way that the discrete solution must converge to a certain extent, say $10^{-6}$. The computations are now performed by using a uniform grid of 1200 points and a time step of $\Delta \tau=10^{-7}$, and are accurate to fourteen decimal places using double-precision arithmetic on PC. Within the thermal boundary layer, approximate 40 grid points are used to calculate the temperature variation with time. With Eq. (17), all physical variables of the gas phase, which are expressed in terms of $T_{\mathrm{s}}^{*}$ and $Y_{\mathrm{v}}(1)$, can then be determined.

\subsection{Stability analysis}

\subsubsection{Perturbed equations}

To determine the onset of instability, situations with infinitesimal disturbances on the basic flow of the droplet are considered. Since all the diffusion time scales of the gas phase are two order smaller than those of the liquid phase and there is no instability mechanism other than the onset of Marangoni instability of the droplet, any disturbance in the gas phase will be damped readily when consideration is mainly on the variation of the liquid phase. Therefore, the perturbation equations of the gas phase are neglected for simplification and its effect on the Marangoni instability of the droplet is directly through the thermal and mass boundary conditions on the interface.

All the quantities $\vec{V}_{1}^{*}, T_{1}^{*}, p_{1}^{*}, Y_{1}^{*}$ are perturbed. In the following analysis, a superscript "'" will be used to denote the perturbed quantities and the subscript 1 , used for the liquid phase previously, is neglected for simplification. After removing the higher order terms and the products of perturbed quantities, the dimensionless equations of the perturbed fields become as follows:

$$
\begin{aligned}
& \nabla \cdot \vec{V}^{\prime}=0, \\
& \left(\nabla^{2}-\frac{1}{P r_{1}} \frac{\partial}{\partial \tau}\right) \vec{V}^{\prime}=\frac{1}{P r_{1}} \nabla p^{\prime}, \\
& \left(\nabla^{2}-\frac{\partial}{\partial \tau}\right) T^{\prime}=\vec{V}^{\prime} \cdot \nabla T^{*}, \\
& \left(\nabla^{2}-L e_{1} \frac{\partial}{\partial \tau}\right) Y^{\prime}=L e_{1} \vec{V}^{\prime} \cdot \nabla Y^{*} .
\end{aligned}
$$


The corresponding boundary conditions are

at $R=0$,

$$
\begin{aligned}
& \vec{V}^{\prime}=\text { finite }, \\
& T^{\prime}=\text { finite }, \\
& Y^{\prime}=\text { finite },
\end{aligned}
$$

and at the interface $(R=1)$,

$$
\begin{aligned}
& u_{R}^{\prime}=\frac{\partial \mathrm{Ev}}{\partial T^{*}} T^{\prime}+\frac{\partial \mathrm{Ev}}{\partial Y^{*}} Y^{\prime}, \\
& R \frac{\partial}{\partial R}\left(\frac{u_{\theta}^{\prime}}{R}\right)+\frac{1}{R} \frac{\partial u_{R}^{\prime}}{\partial \theta}=-\frac{M a_{\mathrm{T}}}{R} \frac{\partial T^{\prime}}{\partial \theta}-\frac{M a_{\mathrm{S}}}{R} \frac{\partial Y^{\prime}}{\partial \theta}, \\
& \frac{1}{R \sin \theta} \frac{\partial u_{R}^{\prime}}{\partial \phi}+R \frac{\partial}{\partial R}\left(\frac{u_{\phi}^{\prime}}{R}\right)=-\frac{M a_{\mathrm{T}}}{R \sin \theta} \frac{\partial T^{\prime}}{\partial \phi}-\frac{M a_{\mathrm{S}}}{R \sin \theta} \frac{\partial Y^{\prime}}{\partial \phi}, \\
& -\frac{\partial T^{\prime}}{\partial R}=\left(B i+\frac{\partial B i}{\partial T^{*}} T^{*}+\frac{\partial Q}{\partial T^{*}}\right) T^{\prime}+\left(\frac{\partial B i}{\partial Y^{*}} T^{*}+\frac{\partial Q}{\partial Y^{*}}\right) Y^{\prime}, \\
& -\frac{1}{L e_{1}} \frac{\partial Y^{\prime}}{\partial R}=\frac{\partial \mathrm{Ev}}{\partial T^{*}}\left(-Y^{*}+\frac{1}{Y_{0}}\right) T^{\prime}-\left(\mathrm{Ev}+\frac{\partial \mathrm{Ev}}{\partial Y^{*}} Y^{*}-\frac{1}{Y_{0}} \frac{\partial \mathrm{Ev}}{\partial Y^{*}}\right) Y^{\prime} .
\end{aligned}
$$

The definitions of the thermal and solute Marangoni numbers are

$$
M a_{\mathrm{T}}=\frac{-(\partial \sigma / \partial T) m^{\prime} h_{\mathrm{lg}} r_{0}^{2}}{\mu_{1} \alpha_{1} k_{1}} \text { and } M a_{\mathrm{S}}=\frac{-\left(\partial \sigma / \partial Y_{1}\right) Y_{0} r_{0}}{\mu_{1} \alpha_{1}},
$$

respectively.

By virtue of the continuity equation, the two tangential stress boundary conditions can be combined into a single one:

$$
\frac{\partial^{2}}{\partial R^{2}}\left(R u_{R}^{\prime}\right)-\frac{\left(2+L^{2}\right)\left(R u_{R}^{\prime}\right)}{R^{2}}=\frac{M a_{\mathrm{T}}}{R} L^{2} T^{\prime}+\frac{M a_{\mathrm{S}}}{R} L^{2} Y^{\prime}
$$

where

$$
L^{2}=\frac{1}{\sin \theta} \frac{\partial}{\partial \theta}\left(\sin \theta \frac{\partial}{\partial \theta}\right)+\frac{1}{\sin ^{2} \theta} \frac{\partial^{2}}{\partial \phi^{2}}=R^{2} \nabla^{2}-\frac{\partial}{\partial R}\left(R^{2} \frac{\partial}{\partial R}\right)
$$

with $\nabla^{2}$ denoting the Laplace operator in the spherical coordinate, i.e.,

$$
\nabla^{2}=\frac{1}{R^{2}} \frac{\partial}{\partial R}\left(R^{2} \frac{\partial}{\partial R}\right)+\frac{1}{R^{2} \sin \theta} \frac{\partial}{\partial \theta}\left(\sin \theta \frac{\partial}{\partial \theta}\right)+\frac{1}{R^{2} \sin ^{2} \theta} \frac{\partial^{2}}{\partial \phi^{2}} .
$$

By eliminating the pressure term in Eq. (32), the perturbed $R$-momentum equation in terms of $u_{R}^{\prime}$ can be derived, i.e.,

$$
\nabla^{2}\left[\nabla^{2}-\frac{1}{P r_{1}} \frac{\partial}{\partial \tau}\right]\left(R u_{R}^{\prime}\right)=0
$$

\subsubsection{The method of normal mode}

Motivated by the operator $L^{2}$, the perturbed quantities are expressed in terms of spherical harmonics, i.e.,

$$
\begin{aligned}
& R u_{R}^{\prime}=U(R) Y_{l}^{m}(\theta, \phi) \mathrm{e}^{\omega \tau}, \\
& T^{\prime}=\mathfrak{J}(R) Y_{l}^{m}(\theta, \phi) \mathrm{e}^{\omega \tau}, \\
& Y^{\prime}=\mathbf{Y}(R) Y_{l}^{m}(\theta, \phi) \mathrm{e}^{\omega \tau},
\end{aligned}
$$

where $l$ and $m$ are integers which are used to distinguish the different modes of disturbances, and $\omega$ represents their time-dependent nature. The spherical harmonics $Y_{l}^{m}$ satisfies the following equation:

$$
\left[\frac{1}{\sin \theta} \frac{\partial}{\partial \theta}\left(\sin \theta \frac{\partial}{\partial \theta}\right)+\frac{1}{\sin ^{2} \theta} \frac{\partial^{2}}{\partial \phi^{2}}\right] Y_{l}^{m}(\theta, \phi)=-l(l+1) Y_{l}^{m}(\theta, \phi),
$$


wherein

$$
Y_{l}^{m}(\theta, \phi)=P_{l}^{m}(\cos \theta) \mathrm{e}^{i m \phi},
$$

and $P_{l}^{m}(\cos \theta)$ are the associated Legendre polynomials.

Substitution of the solution forms given by Eq. (46) into Eqs. (33), (34), and (45) and boundary conditions (35)-(38) and (41)-(43) gives

$$
\begin{aligned}
& {\left[\frac{\mathrm{d}^{2}}{\mathrm{~d} R^{2}}+\frac{2}{R} \frac{\mathrm{d}}{\mathrm{d} R}-\frac{l(l+1)}{R^{2}}\right]\left[\frac{\mathrm{d}^{2}}{\mathrm{~d} R^{2}}+\frac{2}{R} \frac{\mathrm{d}}{\mathrm{d} R}-\frac{l(l+1)}{R^{2}}-\frac{\omega}{P r_{1}}\right] U=0,} \\
& {\left[\frac{\mathrm{d}^{2}}{\mathrm{~d} R^{2}}+\frac{2}{R} \frac{\mathrm{d}}{\mathrm{d} R}-\frac{l(l+1)}{R^{2}}-\omega\right] \mathfrak{J}-\frac{U}{R} \frac{\partial T^{*}}{\partial R}=0,} \\
& {\left[\frac{\mathrm{d}^{2}}{\mathrm{~d} R^{2}}+\frac{2}{R} \frac{\mathrm{d}}{\mathrm{d} R}-\frac{l(l+1)}{R^{2}}-L e_{1} \omega\right] \mathbf{Y}-L e_{1} \frac{U}{R} \frac{\partial Y^{*}}{\partial R}=0,}
\end{aligned}
$$

at $R=0$,

$$
\begin{aligned}
& U=0, \\
& \mathfrak{I}=\text { finite }, \\
& \mathbf{Y}=\text { finite },
\end{aligned}
$$

at $R=1$,

$$
\begin{aligned}
& \frac{U}{R}=\frac{\partial \mathrm{Ev}}{\partial T^{*}} \mathfrak{I}+\frac{\partial \mathrm{Ev}}{\partial Y^{*}} \mathbf{Y}, \\
& \frac{\mathrm{d}^{2} U}{\mathrm{~d} R^{2}}-\frac{2-l(l+1)}{R^{2}} U=-\frac{M a_{\mathrm{T}} l(l+1)}{R} \mathfrak{I}-\frac{M a_{\mathrm{S}} l(l+1)}{R} \mathbf{Y}, \\
& -\frac{\mathrm{d} \mathfrak{I}}{\mathrm{d} R}=\left(B i+\frac{\partial B i}{\partial T^{*}} T^{*}+\frac{\partial Q}{\partial T^{*}}\right) \mathfrak{I}+\left(\frac{\partial B i}{\partial Y^{*}} T^{*}+\frac{\partial Q}{\partial Y^{*}}\right) \mathbf{Y}=\chi_{1} \mathfrak{I}+\chi_{2} \mathbf{Y}, \\
& \frac{1}{L e_{1}} \frac{\mathrm{d} \mathbf{Y}}{\mathrm{d} R}=\frac{\partial \mathrm{Ev}}{\partial T^{*}}\left(Y^{*}-\frac{1}{Y_{0}}\right) \mathfrak{I}+\left(\mathrm{Ev}+\frac{\partial \mathrm{Ev}}{\partial Y^{*}} Y^{*}-\frac{\partial \mathrm{Ev}}{\partial Y^{*}} \frac{1}{Y_{0}}\right) \mathbf{Y}=\zeta_{1} \mathfrak{I}+\zeta_{2} \mathbf{Y} .
\end{aligned}
$$

In the above expressions,

$$
\chi_{1}=B i+\frac{\partial B i}{\partial T^{*}} T^{*}+\frac{\partial Q}{\partial T^{*}} \quad \text { and } \quad \chi_{2}=\frac{\partial B i}{\partial Y^{*}} T^{*}+\frac{\partial Q}{\partial Y^{*}}
$$

are parameters indicating the energy flux due to temperature and concentration fluctuations, respectively;

$$
\zeta_{1}=\frac{\partial \mathrm{Ev}}{\partial T^{*}}\left(Y^{*}-\frac{1}{Y_{0}}\right) \quad \text { and } \zeta_{2}=\left(\mathrm{Ev}+\frac{\partial \mathrm{Ev}}{\partial Y^{*}} Y^{*}-\frac{\partial \mathrm{Ev}}{\partial Y^{*}} \frac{1}{Y_{0}}\right)
$$

are parameters representing the mass flux due to temperature and concentration fluctuations, respectively.

The instability problem is then reduced to an eigenvalue problem consisting of Eqs. (49)-(51) with boundary conditions (52)-(58), which gives, in a functional form,

$$
f\left(\omega, l, M a_{\mathrm{T}}, M a_{\mathrm{S}}, P r_{1}, L e_{1}, B i, \mathrm{Ev}, Q\right)=0 .
$$

In general, the eigenvalue $\omega$ is a complex quantity

$$
\omega=\omega_{r}+\iota \omega_{i} \text {. }
$$

The real part $\omega_{r}$ denotes the degree of amplification or damping, whereas the imaginary part $\omega_{i}$ is the circular frequency of the disturbance. The condition for stability is that $\omega_{r}$ is negative for any value of $l$ and other parameters of the system. Of particular interest is the neutral stability with

$$
\omega_{r}\left(l, M a_{\mathrm{T}}, M a_{\mathrm{S}}, P r_{1}, L e_{1}, B i, \mathrm{Ev}, Q\right)=0 .
$$

This is the condition to be examined in Section 3 for an evaporating binary droplet. 
However, the imaginary part of $\omega\left(\omega_{i}\right)$ will not vanish in general, even if the real part of $\omega\left(\omega_{r}\right)$ does. If $\omega_{r}=0$ implies that $\omega_{i}=0$ for every $l$, it is recognized to have exchange of stabilities and stationary instability will occur. Otherwise, oscillatory instability will occur for certain wave numbers of disturbances. In the present study, only the stationary instability is considered and investigated.

\section{Stationary instability}

\subsection{Criterion for instability}

For the onset of stationary instability, i.e. $\omega=0$, the eigenvalue problem, Eqs. (49)-(51), is simplified to

$$
\begin{aligned}
& {\left[\frac{\mathrm{d}^{2}}{\mathrm{~d} R^{2}}+\frac{2}{R} \frac{\mathrm{d}}{\mathrm{d} R}-\frac{l(l+1)}{R^{2}}\right]^{2} U=0,} \\
& {\left[\frac{\mathrm{d}^{2}}{\mathrm{~d} R^{2}}+\frac{2}{R} \frac{\mathrm{d}}{\mathrm{d} R}-\frac{l(l+1)}{R^{2}}\right] \mathfrak{J}-\frac{U}{R} \frac{\partial T^{*}}{\partial R}=0,} \\
& {\left[\frac{\mathrm{d}^{2}}{\mathrm{~d} R^{2}}+\frac{2}{R} \frac{\mathrm{d}}{\mathrm{d} R}-\frac{l(l+1)}{R^{2}}\right] \mathbf{Y}-L e_{1} \frac{U}{R} \frac{\partial Y^{*}}{\partial R}=0}
\end{aligned}
$$

together with boundary conditions (52)-(58). The solution of Eq. (62), with boundary condition (52), is

$$
U(R)=A\left(R^{l}-a R^{l+2}\right) .
$$

The constant $A$ is arbitrary, depending on the amplitude of the initial disturbances, but it drops out in later analysis [9]. $a$ is a constant to be determined from the condition of continuity of mass flux at the free surface, i.e., Eq. (55).

With the above velocity distribution, the solutions of Eqs. (63) and (64) subject to conditions (57) and (58) are

$$
\begin{aligned}
& \mathfrak{I}(R)=B R^{l}-A \frac{R^{-(l+1)}}{2 l+1} \int_{0}^{R}\left(s^{2 l+1}-a s^{2 l+3}\right) \frac{\partial T^{*}}{\partial s} \mathrm{~d} s+A \frac{R^{l}}{2 l+1} \int_{0}^{R}\left(1-a s^{2}\right) \frac{\partial T^{*}}{\partial s} \mathrm{~d} s, \\
& \mathbf{Y}(R)=C R^{l}-A L e_{1} \frac{R^{-(l+1)}}{2 l+1} \int_{0}^{R}\left(s^{2 l+1}-a s^{2 l+3}\right) \frac{\partial Y^{*}}{\partial s} \mathrm{~d} s+A L e_{1} \frac{R^{l}}{2 l+1} \int_{0}^{R}\left(1-a s^{2}\right) \frac{\partial Y^{*}}{\partial s} \mathrm{~d} s,
\end{aligned}
$$

where

$$
\begin{aligned}
& B=\frac{A b^{-1}}{2 l+1}\left\{I_{1}\left[\left(\chi_{1}-l-1\right)\left(\zeta_{2}-\frac{l}{L e_{1}}\right)-\zeta_{1} \chi_{2}\right]-I_{2} b-I_{3} \chi_{2}(2 l+1)\right\} \\
& C=\frac{A b^{-1}}{2 l+1}\left\{I_{1} \zeta_{1}(2 l+1)+I_{3} L e_{1}\left[\left(\chi_{1}+l\right)\left(\zeta_{2}+\frac{l}{L e_{1}}+\frac{1}{L e_{1}}\right)-\zeta_{1} \chi_{2}\right]-I_{4} L e_{1} b\right\} \\
& b=\left[\left(\chi_{1}+l\right)\left(\zeta_{2}-\frac{l}{L e_{1}}\right)-\zeta_{1} \chi_{2}\right] \\
& I_{1}=\int_{0}^{1}\left(R^{2 l+1}-a R^{2 l+3}\right) \frac{\partial T^{*}}{\partial R} \mathrm{~d} R \\
& I_{2}=\int_{0}^{1}\left(1-a R^{2}\right) \frac{\partial T^{*}}{\partial R} \mathrm{~d} R \\
& I_{3}=\int_{0}^{1}\left(R^{2 l+1}-a R^{2 l+3}\right) \frac{\partial Y^{*}}{\partial R} \mathrm{~d} R \\
& I_{4}=\int_{0}^{1}\left(1-a R^{2}\right) \frac{\partial Y^{*}}{\partial R} \mathrm{~d} R .
\end{aligned}
$$


By condition (55), the constant $a$ can now be determined as

$$
a=\frac{b+\left[\frac{\partial \mathrm{Ev}}{\partial T^{*}}\left(\zeta_{2}-\frac{l}{L e_{1}}\right)-\frac{\partial \mathrm{Ev}}{\partial Y^{*}} \zeta_{1}\right] \int_{0}^{1} R^{2 l+1} \frac{\partial T^{*}}{\partial R} \mathrm{~d} R+\left[\frac{\partial \mathrm{Ev}}{\partial T^{*}} \chi_{2}-\frac{\partial \mathrm{Ev}}{\partial Y^{*}}\left(\chi_{1}+l\right)\right] \int_{0}^{1} R^{2 l+1} \frac{\partial Y^{*}}{\partial R} \mathrm{~d} R}{b+\left[\frac{\partial \mathrm{Ev}}{\partial T^{*}}\left(\zeta_{2}-\frac{l}{L e_{1}}\right)-\frac{\partial \mathrm{Ev}}{\partial Y^{*}} \zeta_{1}\right] \int_{0}^{1} R^{2 l+3} \frac{\partial T^{*}}{\partial R} \mathrm{~d} R+\left[\frac{\partial \mathrm{Ev}}{\partial T^{*}} \chi_{2}-\frac{\partial \mathrm{Ev}}{\partial Y^{*}}\left(\chi_{1}+l\right)\right] \int_{0}^{1} R^{2 l+3} \frac{\partial Y^{*}}{\partial R} \mathrm{~d} R} .
$$

With the perturbed velocity, temperature and concentration fields solved as in Eqs. (65)-(67), the stability curves in $\left(M a_{\mathrm{T}}, M a_{\mathrm{S}}\right)$-plane are then determined by the following relation, which is derived from Eq. (56), i.e.,

$$
M a_{\mathrm{T}}\left[\left(\zeta_{2}-\frac{l}{L e_{1}}\right) I_{1}+\chi_{2} I_{3}\right]-M a_{\mathrm{S}}\left[\zeta_{1} I_{1}+\left(\chi_{1}+l\right) I_{3}\right]=-\left[\left(\chi_{1}+l\right)\left(\zeta_{2}-\frac{l}{L e_{1}}\right)-\zeta_{1} \chi_{2}\right] \frac{2\left[a\left(l^{2}+2 l\right)-l^{2}+1\right]}{l(l+1)} .
$$

Thus, the stability curves in the $\left(M a_{\mathrm{T}}, M a_{\mathrm{S}}\right)$-plane are straight lines.

\subsection{Results and discussion}

Numerical calculations have been performed on Eq. (76) for a binary droplet, $20 \%$ heptane and $80 \%$ hexadecane (percentage is by mass), evaporating in dry air at various initial temperatures. With a much higher boiling point, the volatility of hexadecane is negligible compared to heptane. All calculations assume that the ambient pressure is $1 \mathrm{~atm}$ and the specified time instants are chosen that the quasi-steady assumptions are not violated. The stability calculations require the determination of properties of the droplet and surrounding air. Thermodynamic and transport properties of a heptane-hexadecane droplet are referred to the references [7,24]. To accommodate the variation of gas phase properties, a simple $1 / 3$ rule [14] is used to evaluate the reference temperature and the reference mole fraction of the evaporating component, i.e.,

$$
\begin{aligned}
& T_{\mathrm{r}}=T_{\mathrm{s}}+\frac{1}{3}\left(T_{\infty}-T_{\mathrm{s}}\right), \\
& m_{\mathrm{v}, \text { ref }}=\left.m_{\mathrm{v}}\right|_{R=1}+\frac{1}{3}\left(m_{\mathrm{v}, \infty}-\left.m_{\mathrm{v}}\right|_{R=1}\right) .
\end{aligned}
$$

Moreover, the Mason and Monchick mixture rule [16] is used for the evaluation of the mixture viscosity and the Wilke rule [10] for the mixture thermal conductivity. The thermodynamic properties and molecular weight are calculated by assuming an ideal gas mixture.

Because the linear relationship between $M a_{\mathrm{T}}$ and $M a_{\mathrm{S}}$, the stability curves, as determined by Eq. (76) for each specified wave number $l$, in the $\left(M a_{\mathrm{T}}, M a_{\mathrm{S}}\right)$-plane are straight lines and are shown in Figs. 2 and 3 for a heptanehexadecane droplet with various initial temperatures and at two specific time instants, i.e., $\tau=0.0001$ and $\tau=0.001$. For each stability line, the region below which and bounded by the coordinates is a stable one and the one above which is an unstable region, with respect to the corresponding wave number. The boundary adjacent to the stable region is defined as the stability boundary. The corresponding $M a_{\mathrm{T}}, M a_{\mathrm{S}}$ and $l$ on the stability boundary are defined as critical thermal Marangoni number, critical solute Marangoni number, and critical wave numbers and are indicated by $M a_{\mathrm{T}, \mathrm{C}}$, $M a_{\mathrm{S}, \mathrm{C}}$, and $l_{\mathrm{c}}$. The numerically calculated values are tabulated in Tables 2 and 3.
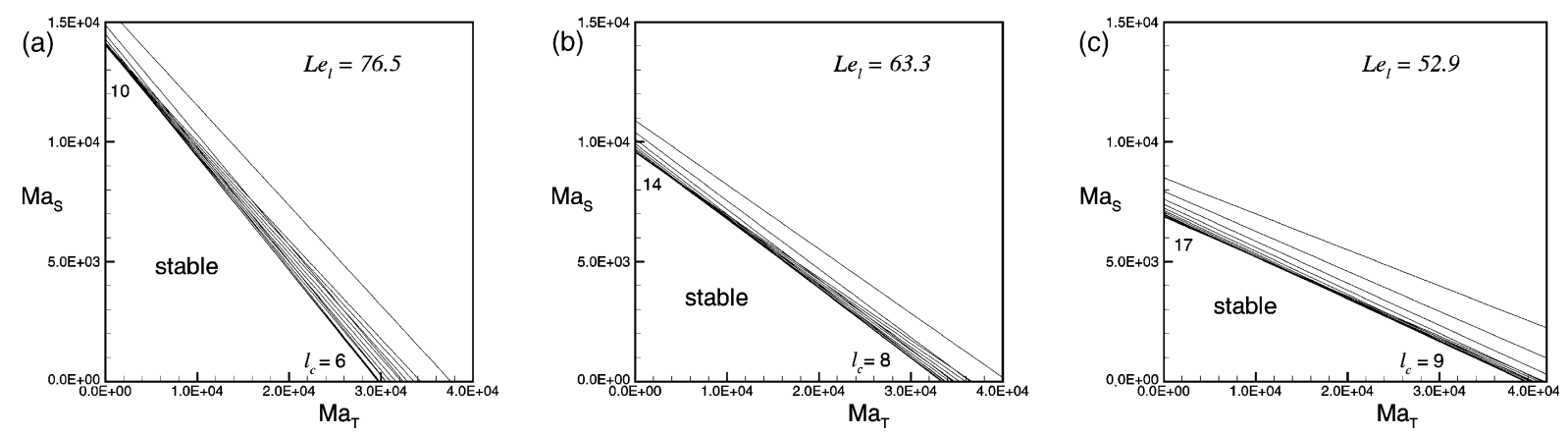

Fig. 2. Stability lines in $\left(M a_{\mathrm{T}}, M a_{\mathrm{S}}\right)$-plane for a heptane-hexadecane droplet with various initial temperatures $\left(Y_{0}=0.2, \tau=0.0001\right)$; each stability line corresponds to a specific wave number: (a) $T_{0}=293 \mathrm{~K}$ (critical wave number, $l_{\mathrm{c}}=6 \sim 10$ ); (b) $T_{0}=303 \mathrm{~K}$ (critical wave number, $l_{\mathrm{c}}=8 \sim 14$ ); (c) $T_{0}=313 \mathrm{~K}$ (critical wave number, $l_{\mathrm{c}}=9 \sim 17$ ). 

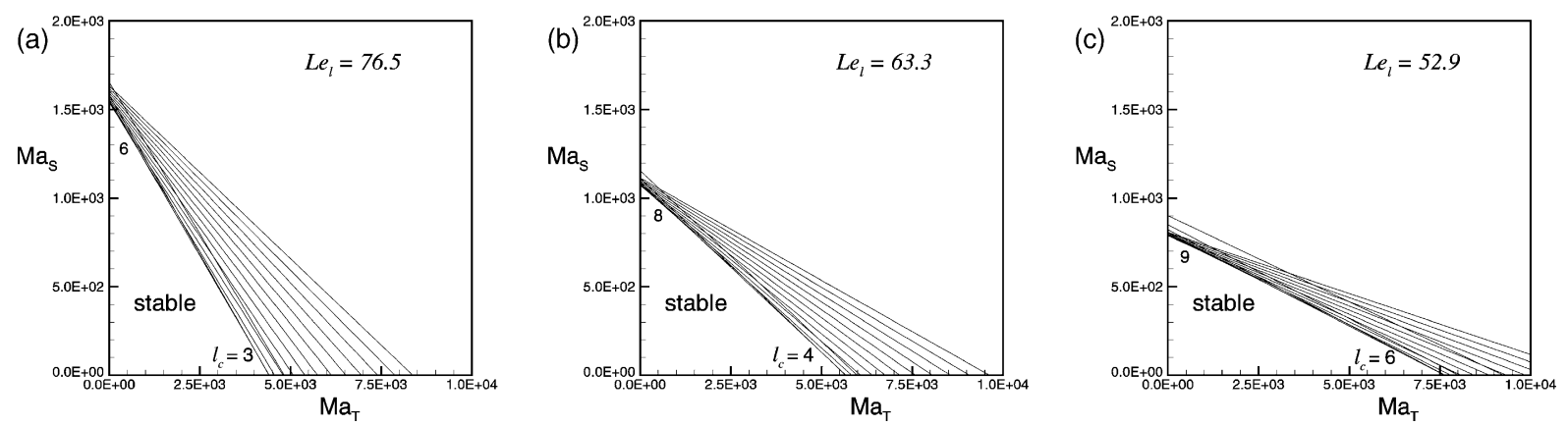

Fig. 3. Stability lines in $\left(M a_{\mathrm{T}}, M a_{\mathrm{S}}\right)$-plane for a heptane-hexadecane droplet with various initial temperatures $\left(Y_{0}=0.2, \tau=0.001\right)$; each stability line corresponds to a specific wave number: (a) $T_{0}=293 \mathrm{~K}$ (critical wave number, $l_{\mathrm{c}}=3 \sim 6$ ); (b) $T_{0}=303 \mathrm{~K}$ (critical wave number, $l_{\mathrm{c}}=4 \sim 8$ ); (c) $T_{0}=313 \mathrm{~K}$ (critical wave number, $l_{\mathrm{c}}=6 \sim 9$ ).

Table 2

Numerically calculated values of $M a_{\mathrm{T}, \mathrm{C}}, M a_{\mathrm{S}, \mathrm{C}}$ and $l_{\mathrm{c}}$ for a heptane-hexadecane droplet at different initial temperatures with $Y_{0}=0.2$ and $\tau=0.0001$

\begin{tabular}{lllr}
\hline$T_{0}$ & $M a_{\mathrm{T}, \mathrm{C}}$ & $M a_{\mathrm{S}, \mathrm{C}}$ & $l_{\mathrm{c}}$ \\
\hline 293 & $2.97 \times 10^{4}-1.67 \times 10^{4}$ & $0-6.19 \times 10^{3}$ & 6 \\
293 & $1.67 \times 10^{4}-6.86 \times 10^{3}$ & $6.19 \times 10^{3}-1.09 \times 10^{4}$ & 7 \\
293 & $6.86 \times 10^{3}-2.99 \times 10^{3}$ & $1.09 \times 10^{4}-1.27 \times 10^{4}$ & 8 \\
293 & $2.99 \times 10^{3}-9.05 \times 10^{2}$ & $1.27 \times 10^{4}-1.36 \times 10^{4}$ & 9 \\
293 & $9.05 \times 10^{2}-0$ & $1.36 \times 10^{4}-1.40 \times 10^{4}$ & 10 \\
303 & $3.33 \times 10^{4}-1.80 \times 10^{4}$ & $0-4.48 \times 10^{3}$ & 8 \\
303 & $1.80 \times 10^{4}-1.03 \times 10^{4}$ & $4.48 \times 10^{3}-6.70 \times 10^{3}$ & 9 \\
303 & $1.03 \times 10^{4}-6.17 \times 10^{3}$ & $6.70 \times 10^{3}-7.86 \times 10^{3}$ & 10 \\
303 & $6.17 \times 10^{3}-3.61 \times 10^{3}$ & $7.86 \times 10^{3}-8.58 \times 10^{3}$ & 11 \\
303 & $3.61 \times 10^{3}-1.82 \times 10^{3}$ & $8.58 \times 10^{3}-9.08 \times 10^{3}$ & 12 \\
303 & $1.82 \times 10^{3}-4.99 \times 10^{2}$ & $9.08 \times 10^{3}-9.43 \times 10^{3}$ & 13 \\
303 & $4.99 \times 10^{2}-0$ & $9.43 \times 10^{3}-9.57 \times 10^{3}$ & 9 \\
313 & $3.77 \times 10^{4}-3.42 \times 10^{4}$ & $0-6.45 \times 10^{2}$ & 10 \\
313 & $3.42 \times 10^{4}-2.02 \times 10^{4}$ & $6.45 \times 10^{2}-3.24 \times 10^{3}$ & 1 \\
313 & $2.02 \times 10^{4}-1.31 \times 10^{4}$ & $3.24 \times 10^{3}-4.54 \times 10^{3}$ & 1 \\
313 & $1.31 \times 10^{4}-8.82 \times 10^{3}$ & $4.54 \times 10^{3}-5.32 \times 10^{3}$ & 13 \\
313 & $8.82 \times 10^{3}-5.90 \times 10^{3}$ & $5.32 \times 10^{3}-5.85 \times 10^{3}$ & 14 \\
313 & $5.90 \times 10^{3}-3.76 \times 10^{3}$ & $5.85 \times 10^{3}-6.22 \times 10^{3}$ & 15 \\
313 & $3.76 \times 10^{3}-2.11 \times 10^{3}$ & $6.22 \times 10^{3}-6.51 \times 10^{3}$ & 16 \\
313 & $2.11 \times 10^{3}-7.85 \times 10^{2}$ & $6.51 \times 10^{3}-6.73 \times 10^{3}$ & 17 \\
313 & $7.85 \times 10^{2}-0$ & $6.73 \times 10^{3}-6.86 \times 10^{3}$ & \\
\hline
\end{tabular}

Several very interesting and important aspects can be learned from Figs. 2 and 3. They are: (1) Since, for a heptanehexadecane droplet, the thermocapillary effect and diffuso-capillary effect augment each other, the stability lines possess negative slopes which means, with a stronger diffuso-capillary effect, the critical thermal Marangoni number, $M a_{\mathrm{T}, \mathrm{C}}$ becomes smaller and vice versa. (2) When the diffuso-capillary effect is negligibly small, i.e. $M a_{\mathrm{S}} \approx 0, M a_{\mathrm{T}, \mathrm{C}}$ increases with the initial temperature of the droplet and decrease as time proceeds, which are consistent with the results obtained by Ha [11] and Ha and Lai [12]. The explanation for this phenomenon as given by the authors is repeated as follows. There are two competing mechanisms determining the onset of instability. As stated previously the temperature reduction near the free surface is the driving potential for the Marangoni instability. Therefore, with a higher initial temperature which results in a larger temperature reduction near the free surface, the droplet on one hand should become more unstable. However, the heat transfer to the surrounding gas due to evaporation, which increases with initial temperature, possesses the tendency to smooth the surface temperature disturbances. Therefore, the droplet, on the other hand, becomes more stable with a higher initial temperature. The competition between these two mechanisms will finally determine the effect of the initial temperature on the droplet instability. (3) When the thermocapillary effect is 
Table 3

Numerically calculated values of $M a_{\mathrm{T}, \mathrm{C}}, M a_{\mathrm{S}, \mathrm{C}}$ and $l_{\mathrm{c}}$ for a heptane-hexadecane droplet at different initial temperatures with $Y_{0}=0.2$ and $\tau=0.001$

\begin{tabular}{llll}
\hline$T_{0}$ & $M a_{\mathrm{T}, \mathrm{C}}$ & $M a_{\mathrm{S}, \mathrm{C}}$ & $l_{\mathrm{c}}$ \\
\hline 293 & $4.41 \times 10^{3}-4.30 \times 10^{3}$ & $0-4.0 \times 10^{1}$ & 3 \\
293 & $4.30 \times 10^{3}-7.77 \times 10^{2}$ & $4.0 \times 10^{1}-1.28 \times 10^{3}$ & 4 \\
293 & $7.77 \times 10^{2}-1.19 \times 10^{2}$ & $1.28 \times 10^{3}-1.50 \times 10^{3}$ & 5 \\
293 & $1.19 \times 10^{2}-0$ & $1.50 \times 10^{3}-1.54 \times 10^{3}$ & 6 \\
303 & $5.66 \times 10^{3}-5.19 \times 10^{3}$ & $0-9.1 \times 10^{1}$ & 4 \\
303 & $5.19 \times 10^{3}-1.56 \times 10^{3}$ & $9.1 \times 10^{1}-7.90 \times 10^{2}$ & 5 \\
303 & $1.56 \times 10^{3}-5.90 \times 10^{2}$ & $7.90 \times 10^{2}-9.69 \times 10^{2}$ & 6 \\
303 & $5.90 \times 10^{2}-1.05 \times 10^{2}$ & $9.69 \times 10^{2}-1.06 \times 10^{3}$ & 7 \\
303 & $1.05 \times 10^{2}-0$ & $1.06 \times 10^{3}-1.07 \times 10^{3}$ & 8 \\
313 & $7.63 \times 10^{3}-2.77 \times 10^{3}$ & $0-5.13 \times 10^{2}$ & 6 \\
313 & $2.77 \times 10^{3}-1.25 \times 10^{3}$ & $5.13 \times 10^{2}-6.68 \times 10^{2}$ & 7 \\
313 & $1.25 \times 10^{3}-4.84 \times 10^{2}$ & $6.68 \times 10^{2}-7.43 \times 10^{2}$ & 8 \\
313 & $4.84 \times 10^{2}-0$ & $7.43 \times 10^{2}-7.88 \times 10^{2}$ & 9 \\
\hline
\end{tabular}

negligible, i.e., $M a_{\mathrm{T}} \approx 0, M a_{\mathrm{S}, \mathrm{C}}$ decreases as the initial temperature of the droplet increases and as time proceeds. This is because the concentration reduction of heptane near the free surface becomes larger when the droplet possesses a higher initial temperature or as time proceeds. Since $M a_{\mathrm{T}} \approx 0$, i.e., the thermocapillary effect is not important, the smoothing effect of evaporation as discussed in item (2) will not effectively delay the occurrence of Marangoni instability. (4) All the stability boundaries are composed of stability lines with different critical wave numbers. The critical wave number, $l_{\mathrm{c}}$, possesses a larger value when the diffuso-capillary effect is dominant, i.e., at larger $M a_{\mathrm{S}, \mathrm{C}}$ and smaller $M a_{\mathrm{T}, \mathrm{C}}$. When the thermocapillary effect is the dominant one, i.e., at larger $M a_{\mathrm{T}, \mathrm{C}}$ and smaller $M a_{\mathrm{S}, \mathrm{C}}, l_{\mathrm{c}}$ has a smaller value. The permissible values of $l_{\mathrm{c}}$ on the stability boundary for different ranges of $\left(M a_{\mathrm{T}}, M a_{\mathrm{S}}\right)$ can be readily found from Tables 2 and 3. Fig. 4, as an example, gives a close look of the stability boundary for $T_{0}=313 \mathrm{~K}$ and $\tau=0.001$. The reason is as follows. Since the Lewis number of a heptane-hexadecane mixture is about 50-80 depending on the initial temperature of the droplet, the solute boundary layer is smaller than the thermal boundary layer near the free surface. Therefore, when the diffuso-capillary effect is dominant, the disturbances for the onset of instability should possess a smaller wavelength or a larger wave number in proportion to, in a certain sense, the solute boundary layer thickness near the free surface. With the same reasoning, when the thermocapillary effect is dominant, $l_{\mathrm{c}}$ should have a smaller value. Moreover, since both the thermal and solute boundary layer thicknesses increase as time proceeds, $l_{\mathrm{c}}$ has smaller values for $\tau=0.001$ than for $\tau=0.0001$.

In order to demonstrate the Lewis-number effect on the onset of Marangoni instability, calculations with $L e_{1}=10,1$, and 0.1 , which are mathematically interesting only, and the one for a heptane-hexadecane droplet, are intentionally shown in Fig. 5 for $T_{0}=293 \mathrm{~K}$ and $\tau=0.0001$. The numerically calculated values of $M a_{\mathrm{T}, \mathrm{C}}, M a_{\mathrm{S}, \mathrm{C}}$ and $l_{\mathrm{c}}$ are tabulated

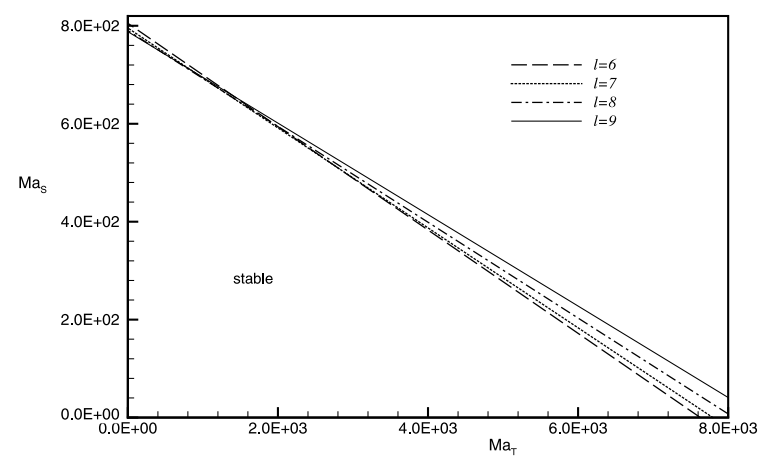

Fig. 4. Stability boundary in $\left(M a_{\mathrm{T}}, M a_{\mathrm{S}}\right)$-plane for a heptane-hexadecane droplet at $T_{0}=313 \mathrm{~K}, Y_{0}=0.2$, and $\tau=0.001$ with $L e_{1}=52.9$; constructed by stability lines with different wave number. 

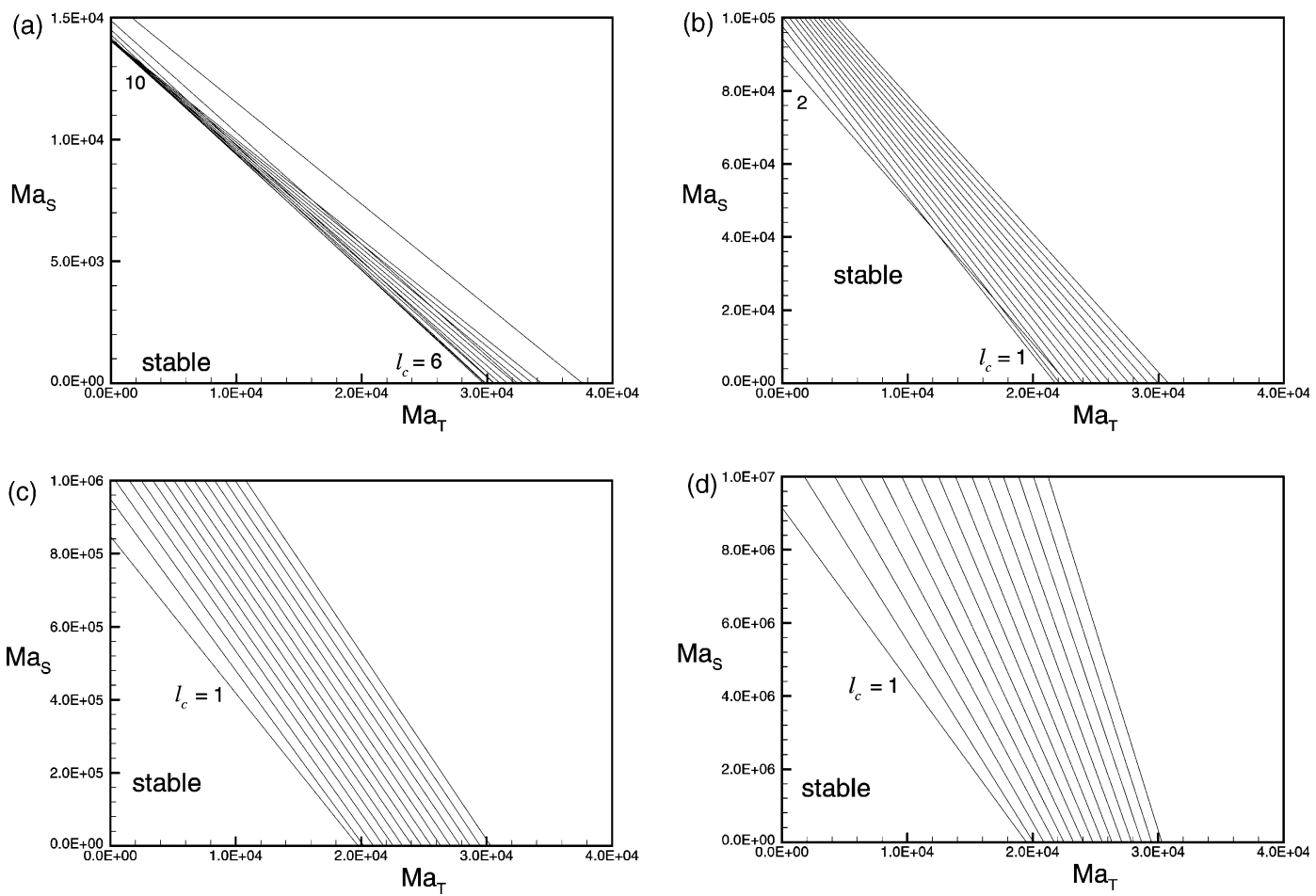

Fig. 5. Lewis number effect on stability lines in $\left(M a_{\mathrm{T}}, M a_{\mathrm{S}}\right)$-plane for evaporating binary droplets at $T_{0}=293 \mathrm{~K}, Y_{0}=0.2$, and $\tau=0.0001$ : (a) $L e_{1}=76.5$ (heptane-hexadecane droplet; $\left.l_{\mathrm{c}}=6-10\right)$; (b) $L e_{1}=10\left(l_{\mathrm{c}}=1-2\right)$; (c) $L e_{1}=1\left(l_{\mathrm{c}}=1\right)$; (d) $L e_{1}=0.1\left(l_{\mathrm{c}}=1\right)$.

Table 4

Numerically calculated values of $M a_{\mathrm{T}, \mathrm{C}}, M a_{\mathrm{S}, \mathrm{C}}$ and $l_{\mathrm{c}}$ for evaporating binary droplets at different Lewis numbers with $T_{0}=293 \mathrm{~K}$, $Y_{0}=0.2$ and $\tau=0.0001$

\begin{tabular}{llll}
\hline$L e_{l}$ & $M a_{\mathrm{T}, \mathrm{C}}$ & $M a_{\mathrm{S}, \mathrm{C}}$ & $l_{\mathrm{c}}$ \\
\hline 76.5 & $2.97 \times 10^{4}-1.67 \times 10^{4}$ & $0-6.19 \times 10^{3}$ & 6 \\
76.5 & $1.67 \times 10^{4}-6.86 \times 10^{3}$ & $6.19 \times 10^{3}-1.09 \times 10^{4}$ & 7 \\
76.5 & $6.86 \times 10^{3}-2.99 \times 10^{3}$ & $1.09 \times 10^{4}-1.27 \times 10^{4}$ & 8 \\
76.5 & $2.99 \times 10^{3}-9.05 \times 10^{2}$ & $1.27 \times 10^{4}-1.36 \times 10^{4}$ & 9 \\
76.5 & $9.05 \times 10^{2}-0$ & $1.36 \times 10^{4}-1.40 \times 10^{4}$ & 10 \\
10 & $2.27 \times 10^{4}-1.22 \times 10^{4}$ & $0-4.16 \times 10^{4}$ & 1 \\
10 & $1.22 \times 10^{4}-0$ & $4.16 \times 10^{4}-9.44 \times 10^{4}$ & 2 \\
1 & $1.98 \times 10^{4}-0$ & $0-8.47 \times 10^{5}$ & 1 \\
0.1 & $1.95 \times 10^{4}-0$ & $0-9.15 \times 10^{6}$ & 1 \\
\hline
\end{tabular}

in Table 4. Similar results are also obtained for $T_{0}=303$ and $313 \mathrm{~K}$ at $\tau=0.001$. The basic phenomena illustrated in the previous paragraph are observed for all the Lewis numbers calculated.

Studying the figures carefully, it can be found that, since the solute boundary layer thickness becomes larger as $L e_{1}$ decreases, $l_{\mathrm{c}}$ becomes smaller correspondingly. Moreover, since the solute boundary layer thickness becomes compatible with the thermal boundary layer thickness as $L e_{1}$ approaches to unity, $l_{\mathrm{c}}$ tends to possess a single value. As a result, when $L e_{1} \leqslant 1, l_{\mathrm{c}}=1$ always. The permissible values of $l_{\mathrm{c}}$ on the stability boundary for different ranges of $\left(M a_{\mathrm{T}}, M a_{\mathrm{S}}\right)$ can be readily found from Table 4 . In addition, $L e_{1}$ has a stronger effect on $M a_{\mathrm{S}, \mathrm{C}}$ than on $M a_{\mathrm{T}, \mathrm{C}}$. From Fig. 5 , it can be seen that when the thermocapillary effect is dominant or $M a_{\mathrm{S}}$ is very small, with the aid of diffuso-capillary effect, $M a_{\mathrm{T}, \mathrm{C}}$ tends to decrease as $L e_{1}$ decreases. While, when the diffuso-capillary effect is dominant or $M a_{\mathrm{T}}$ is very small, $M a_{\mathrm{S}, \mathrm{C}}$ tends to increase drastically as $L e_{1}$ decreases because of the much stronger $L e_{1}$-effect on solute diffusion, as illustrated by Fig. 6. The variation of $M a_{\mathrm{T}, \mathrm{C}}$ with $L e_{1}$ is much milder than that of $M a_{\mathrm{S}, \mathrm{C}}$. 

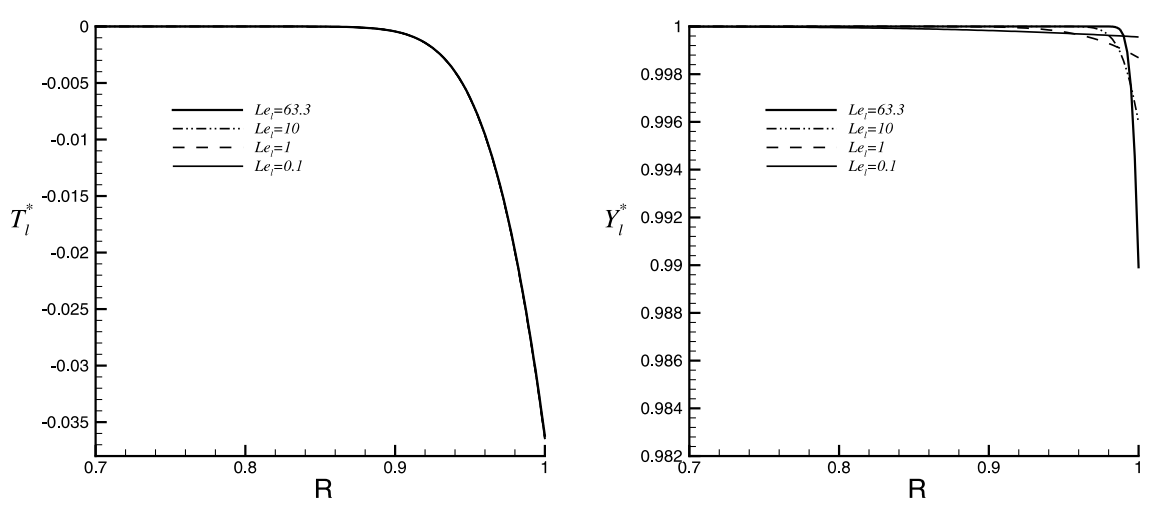

Fig. 6. Lewis-number effect on the dimensionless temperature and mass fraction distributions near the free surface of an evaporating binary droplet at $T_{0}=303 \mathrm{~K}, Y_{0}=0.2$, and $\tau=0.001$.

\section{Conclusions}

The stationary instability of an evaporating binary droplet is investigated. With the quasi-steady approximation which assumes the size change of the droplet is negligible, the surrounding gas motion is asymptotically steady, and the temperature and concentration distribution of the droplet is temporarily frozen at each specified instant, the onset condition for stationary Marangoni instability is obtained through linear stability analysis.

By assuming the surface tension is a monotonically decreasing function of both temperature and solute concentration, the thermocapillary and diffuso-capillary effects augment each other. Therefore, the theoretical analysis predicts a linear relation, with a negative slope, between the onset thermal Marangoni number, $M a_{\mathrm{T}}$, and the onset solute Marangoni number, $M a_{\mathrm{S}}$. Moreover, when $L e_{1}>1$ the critical wave number, $l_{\mathrm{c}}$, may possess different values depending on the variation of thermocapillary effect and diffuso-capillary effect. In addition, as $L e_{1}$ decreases, the critical thermal Marangoni number, $M a_{\mathrm{T}, \mathrm{C}}$, decreases mildly while the critical solute Marangoni number, $M a_{\mathrm{S}, \mathrm{C}}$, increases drastically.

Depending on the relation between the surface tension and temperature or concentration, and the effect on the variations of the surface temperature and local boiling points due to changes in droplet surface composition, the thermocapillary and diffuso-capillary effects may oppose each other [2]. When such a situation occurs, depending on the relative importance between the thermocapillary and diffuso-capillary effects, the onset of Marangoni instability becomes much more complicated and different. As an example, the stability lines in Fig. 2 and 3 may possess positive slopes. A detailed study of the Marangoni instability of an evaporating droplet when the thermocapillary and diffusocapillary effects oppose each other needs more effort and is now under investigation.

\section{Acknowledgements}

The authors thank the National Science Council, Taiwan, ROC, for their financial support of this work through Grant No. NSC88-2212-E-002-028.

\section{References}

[1] I. Aharon, B.D. Shaw, Marangoni instability of bi-component droplet gasification in reduced gravity, in: The 1995 Fall Meeting of the Western States Section of the Combustion Institute, Stanford University, Stanford, CA, 1995, pp. 1-24.

[2] I. Aharon, B.D. Shaw, Marangoni instability of bi-component droplet gasification, Phys. Fluids A 8 (1996) $1820-1827$.

[3] P.L.T. Brian, J.R. Ross, The effect of gibbs adsorption on Marangoni instability in penetration mass transfer, Am. Inst. Chem. Eng. J. 18 (1972) 582-591.

[4] C.F. Chen, Surface tension effects on the onset of double-diffusive convection, in: H.J. Rath (Ed.), Microgravity Fluid Dynamics, 1992, pp. 325-333.

[5] C.F. Chen, T.F. Su, Effect of surface tension on the onset of convection in a double-diffusive layer, Phys. Fluids A 14 (1992) 23602367.

[6] A. Crespo, A. Linan, Unsteady effects in droplet evaporation and combustion, Combustion Sci. Tech. 11 (1975) 9-18. 
[7] T.E. Dauber, R.P. Danner, Data Compilation Tables of Properties of Pure Compounds, New York, 1985.

[8] S.H. Davis, Buoyancy-surface tension instability by the method of energy, J. Fluid Mech. 39 (1969) $347-359$.

[9] H.A. Dijkstra, A.I. van de Vooren, Initial flow development due to Marangoni convection in a mass transfer system, Int. J. Heat and Mass Transfer 28 (1985) 2315-2322.

[10] D.K. Edwards, V.E. Denny, A.F. Mills, in: Transfer Processes, McGraw-Hill, 1973, p. 240.

[11] V.M. Ha, The analysis of Marangoni instability of evaporating liquid, Ph.D. Dissertation, Department of Mechanical Engineering, National Taiwan University, 1998.

[12] V.M. Ha, C.L. Lai, The onset of Stationary Marangoni instability of an evaporating droplet, Proceedings of the Royal Society of London Series A 457 (2001) 885-909.

[13] G.M. Homsy, Global stability of time-dependent flows: impulsively heated or cooled fluid layers, J. Fluid Mech. 60 (1973) 129139.

[14] G.L. Hubbard, V.E. Denny, A.F. Mills, Droplet evaporation: effects of transients and variable properties, Int. J. Heat and Mass Transfer 18 (1974) 1003-1008.

[15] D. Lozinski, M. Matalon, Thermocapillary motion in a spinning vaporizing droplet, Physics of Fluids A 5 (1993) $1596-1601$.

[16] E.A. Mason, L. Monchick, Transport properties of polar-gas mixtures, J. Chem. Phys. 36 (1962) 2746-2757.

[17] C.L. McTaggart, Convection driven by concentration- and temperature-dependent surface tension, J. Fluid Mech. 134 (1983) 301310.

[18] G.P. Neitzel, Onset of convection in impulsively heated or cooled fluid layers, Phys. Fluids 25 (1982) $210-211$.

[19] B.D. Niazmand, H.A. Shaw, H.A. Dwyer, I. Aharaon, Effects of Marangoni convection on transient droplet evaporation, Combustion Sci. Technol. 103 (1995) 219-233.

[20] D.A. Nield, Surface tension and buoyancy effects in cellular convection, J. Fluid Mech. 19 (1964) 341-352.

[21] A. Orell, J.W. Westwater, Spontaneous interfacial cellular convection accompanying mass transfer: ethylene glycol-acetic acidethyl acetate, Am. Inst. Chem. Eng. J. 8 (1962) 350-356.

[22] H.J. Palmer, The hydrodynamic stability of rapidly evaporating liquids at reduced pressure, J. Fluid Mech. 75 (1976) $487-511$.

[23] J.R.A. Pearson, On convection cells induced by surface tension, J. Fluid Mech. 4 (1958) 489-500.

[24] R.C. Reid, J.M. Prausnitz, B.E. Poling, The Properties of Gases and Liquids, McGraw-Hill, 1987.

[25] A.Ye. Rednikov, V.N. Kourdiumov, Yu.S. Ryazantsev, M.G. Velarde, The role of time-varying gravity on motion of a drop induced by Marangoni instability, Phys. Fluids A 7 (1995) 2670-2678.

[26] L.E. Scriven, C.V. Sternling, On cellular convection driven by surface-tension gradients: effects of mean surface tension and surface viscosity, J. Fluid Mech. 19 (1964) 321-340.

[27] S. Slavtchev, V. Naidenov, Z.H. Kozhoukharova, Stationary Marangoni instability in a liquid layer due to non-isothermal gas absorption, in: H.J. Rath (Ed.), Microgravity Fluid Dynamics, 1992, pp. 91-98.

[28] K.A. Smith, On convective instability induced by surface-tension gradients, J. Fluid Mech. 24 (1966) $401-414$.

[29] C.V. Sternling, L.E. Scriven, Interfacial turbulence: hydrodynamic instability and the Marangoni effect, Am. Inst. Chem. Eng. J. 5 (1959) 514-523.

[30] J. Tanny, C.C. Chen, C.F. Chen, Effects of interaction between Marangoni and double-diffusive instabilities, J. Fluid Mech. 303 (1995) 1-21.

[31] A. Vidal, A. Acrivos, Effect of nonlinear temperature profiles on the onset of convection driven by surface tension gradients, Ind. Eng. Chem. Fundam. 7 (1968) 53-58.

[32] S. Wahal, A. Bose, Rayleigh-Benard and interfacial instabilities in two immiscible liquid layers, Phys. Fluids 31 (1988) $3502-3510$.

[33] R.W. Zeren, W.C. Reynolds, Thermal instabilities in two-fluid horizontal layers, J. Fluid Mech. 53 (1972) $305-327$. 